\title{
MORPHOLOGICAL FILTER FOR LOSSLESS IMAGE SUBSAMPLING *
}

\author{
Josep R. Casas, Luis Torres \\ Dept. of Signal Theory and Communications \\ Universitat Politècnica de Catalunya \\ P.O. Box 30.002 - 08071 Barcelona, Spain
}

\begin{abstract}
In this paper, we present a morphological filter for lossless image subsampling for a given downsamplingupsampling strategy. This filter is applied in a multiresolution decomposition and results in a more efficient scheme for image coding purposes than other lossy sampling schemes. Its main advantage is a greatly reduced computational load compared to multiresolution schemes performed with linear filters.
\end{abstract}

\section{INTRODUCTION}

In multiresolution image processing [1] a complete description of an image is obtained by analysis over a whole range of spatial scales. One of the most useful structures for multiscale analysis is a set of successive images, each one being a filtered and subsampled copy of its predecessor. The inherent decomposition (that of the filtering residues or level-to-level differences) is a nonredundant organization of data which has proven to be an efficient tool for coding purposes.

The subsampling step is of prime importance for a good rendition of such coding schemes. At each level, a simplification filter is applied before downsampling, so that the image components that can not be correctly represented in the subsampled image at the next level are removed. The filtering stage actually performs a decomposition of the original image into a simplified version, which may be downsampled, and a residual image (fig. 1). The successive application of such decomposition results in the piramidal multiresolution scheme. If the residues can also be downsampled, then the decomposition is called a subband multiresolution scheme.

In the linear case [2,3], the simplification filter is designed considering frequency criteria. According to the Nyquist theorem, an ideal low-pass filter is required to avoid aliasing effects. However, practical filters do not allow lossless subsampling due to their non-ideal fre-

\footnotetext{
* This work has been partially supported within the RACE Project MORPHECO of the European Union.
}

quency response. A certain amount of aliasing is introduced by remaining frequency components in the stop band, while desired image components suffer some attenuation in the pass band. QMF filters were proposed to cancel out the aliasing effect of the filter transition band under some particular conditions $[4,3]$.

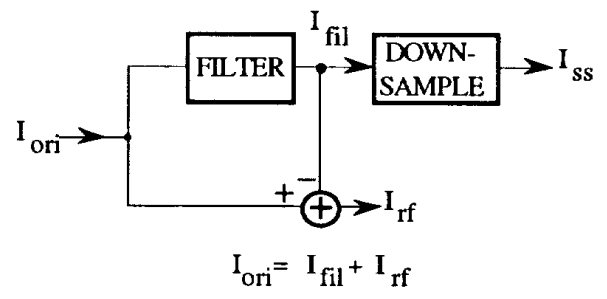

Fig. 1. Decomposition performed by the simplification filter

Non-linear filtering techniques have introduced a different approach in multiscale analysis. Relying on the principle that different resolution levels are perceived as objects of different sizes by the human visual system, size analysis is assumed to perform better than frequency analysis in order to obtain a visual-like decomposition. Mathematical Morphology allows this kind of geometrical analysis of the image components. Morphological filters [5] are increasing and idempotent operators that perform as "sieves" for size and shape features. In addition, they preserve inclusion, which is much closer to the subjective perception of occlusion than the additivity property in the linear case.

Morphological sampling has been formalized in $[6,7]$ and used for coding applications in [8,9]. Opening, closing or a combination of both filters are applied for the simplification stage. They remove from the original image bright or dark components smaller than the new grid spacing, in order to ensure preservation of the remaining components after downsampling. Nevertheless, as happens in the linear case, these morphological filters do not allow transparent (lossless) subsampling either.

The target of this paper is to show that the use of a morphological lossless subsampling scheme yields better 
results for coding purposes than other linear or non-linear lossy sampling schemes.

The paper is organized as follows. Next section analyzes how subsampling losses are dealt with in multiresolution decompositions for image coding. The morphological filter for lossless image subsampling is presented in section 3 and applied to image coding in section 4 . Finally, the coding results are discussed in the last section of the paper.

\section{LOSSY SUBSAMPLING SCHEMES}

Both linear and morphological techniques introduce losses in subsampling schemes. The filtered image can not be exactly reconstructed from its downsampled version trough any interpolation procedure. A limited amount of uncertainty in the interpolated image appears in the two cases but, while in the linear case this uncertainty affects the amplitude values (to a limited extent depending on the non-ideal response of the filter), in the morphological case the uncertainty appears as a positional inaccuracy bounded by the grid spacing at the lower level [6].

For coding purposes, and in order to obtain a lossless decomposition, subsampling losses should be considered together with the filtering residues. For this reason, the interpolation procedure is also introduced in the analysis stage, as shown in fig. 2. If subsampling losses were not considered -as with the scheme presented in fig. 1- only a limited number of successive levels could be computed without noticeable errors in the reconstructed image. On the contrary, if these losses are considered, the coding of the decomposition becomes more difficult due to an increase in the entropy of the residual images at the different levels of the decomposition [9].

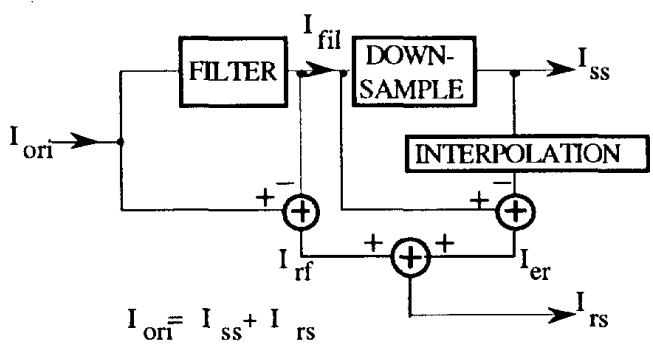

Fig. 2. Lossless decomposition strategy for a lossy subsampling step

Notice that the structure depicted in fig. 2 is of practical use but, somehow, in contradiction with the initial concept of an ideal subsampling step. Actually, the simplification filter is not able to perform the required control over the information -noted by $I_{s s}$ in fig. 2 - that would be correctly represented in the subsampled image and the information that would be not: $I_{r s}$. Therefore, part of the information content in the residual image $\left(\mathrm{I}_{\mathrm{fil}}-\mathrm{I}_{\mathrm{ss}}\right)$ will not be under the control of the filter. In some cases [10], this information loss, $\mathrm{I}_{\mathrm{er}}$, due to the aliasing effect in the downsampling/upsampling step may be even more important than the information contained in the filtering residue, $\mathrm{I}_{\mathrm{rf}}$.

\section{MORPHOLOGICAL FILTER FOR LOSSLESS SUBSAMPLING}

Let us consider a downsampling-upsampling strategy, where the downsampling step is assumed to take every even grid sample from the filtered signal, while upsampling is performed by zero padding and morphological dilation. As the downsampling step is space-variant with the grid position, the morphological filter for simplification will also be variant in space. The basic filtering operator $\xi$ for the one-dimensional signal ' $x$ ' taking values in the discrete locations ' $k$ ' is defined as follows:

$$
\xi\left(x_{k}\right)=\left\{\begin{array}{cl}
\varepsilon_{A}\left(x_{k}\right) & \text { if } k=2 i \\
\varepsilon_{A} *\left(x_{k}\right) & \text { if } k=2 i+1
\end{array}\right.
$$

where $\varepsilon$ stands for morphological erosion and $A$ is a two pixels wide flat structuring element $\left(A^{*}\right.$ is its symmetric).

The filter $\xi$ may be characterized as an erosion because it commutes with the 'inf'(1) operator. However, it is also idempotent and thus it is an algebraic opening, i.e. an increasing, idempotent and anti-extensive operator, that is a morphological filter [5]. Its idempotency and increasing properties can been easily proven. The dual filter $\zeta$, based on a space-variant dilation may also be defined:

$$
\zeta\left(x_{k}\right)=\left\{\begin{aligned}
\delta_{A}\left(x_{k}\right) & \text { if } k=2 i \\
\delta_{A^{*}}\left(x_{k}\right) & \text { if } k=2 i+1
\end{aligned}\right.
$$

Moreover, the definition can be extended to twodimensional signals by separability. For image functions, the filters $\xi$ and $\zeta$ are able to remove those components from the original image that would be lost after downsampling because they are smaller than the new sampling interval. The dilation reconstruction of the filtered and downsampled image using the same structuring element gives exactly the filtered image, $\xi(x)$ or $\zeta(x)$, without any amplitude or positional inaccuracy. Therefore, the decomposition performed by these filters is lossless and the information present in the residual image is accurately controlled by the filtering stage.

The successive application of such subsampling step results in a decomposition of the image where the series of filtering residues contain the progressive differences to the smallest value in a square neighbourhood of side equal to two raised to the times that the basic step (filter $\xi$

(1) Note: The 'inf operator and the morphological erosion ' $\varepsilon$ ' and dilation ' $\delta$ ' are the usual definitions of [4]. 
followed by downsampling) has been applied to the original image. If the filter $\zeta(x)$ is used, the residues will contain the differences to the largest value. Fig. 3 shows the block diagram of the size-multiresolution scheme for a lossless subsampling step.

\section{APPLICATION TO IMAGE CODING}

Apart from the interesting fact of being simultaneously an erosion (dilation) and opening (closing) operator, the presented filter $\xi$ (or $\zeta$ ) is a useful tool for coding applications involving subsampling steps. In fig. 4 we have applied a subband decomposition scheme based on the filter $\xi$ to the original image "Lenna". In order to downsample the residual images, two properties of the filter have been taken into account. The filtering residual is an unsigned function, due to the extensivity property. In addition, as morphological operators do not introduce new image values, a zero value of the residue is found for every pair of grid samples. Therefore, the sign has been used to code the position information of the non-zero residue sample: positive for increasing transitions in the directions left to right and top to bottom.

The morphological decomposition can be compared to the linear case shown in fig. 5, where an 8-tap QMF filter has been used for the simplification step. It can be observed that the residual images in the morphological case are less noisy. As mentioned before, the filter follows a size criterion, so that the components smaller than the size of the new grid are present in the residual image. These components are mainly amplitude variations of 1 pixel size in visual edges and also small maxima or minima of the image. In figs. 6 and 7, the result of two iterative applications of the linear and morphological subband coding scheme is presented.

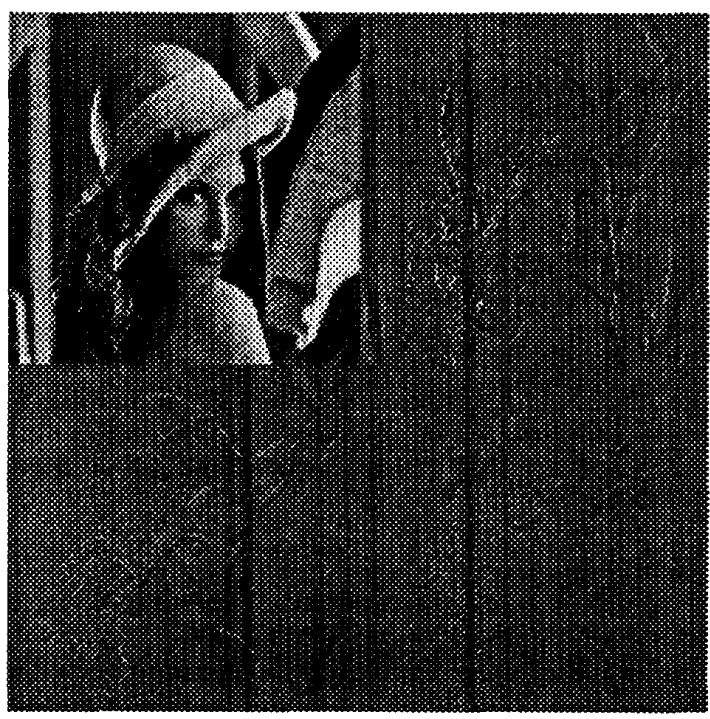

Fig. 4. Morphological subband decomposition ( $\xi$ filter)

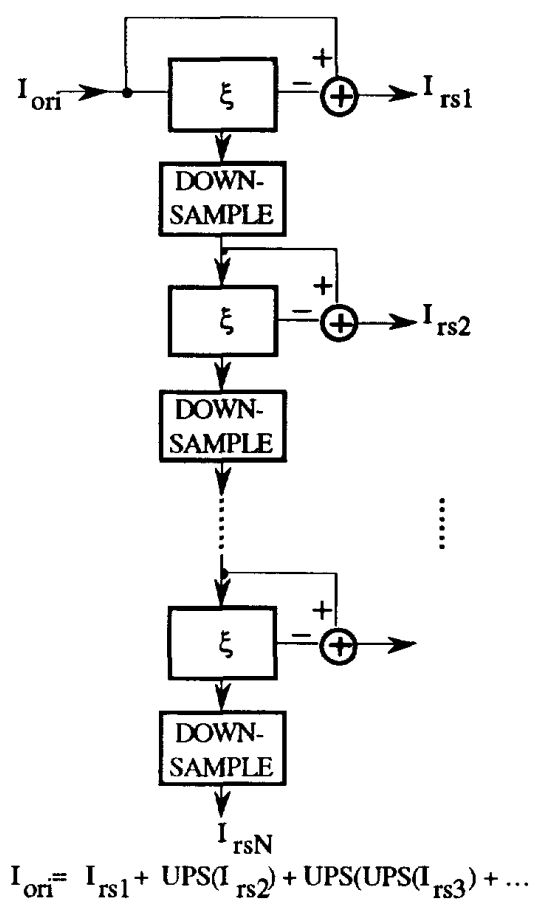

Fig. 3. Multiresolution decomposition with a lossless subsampling step.

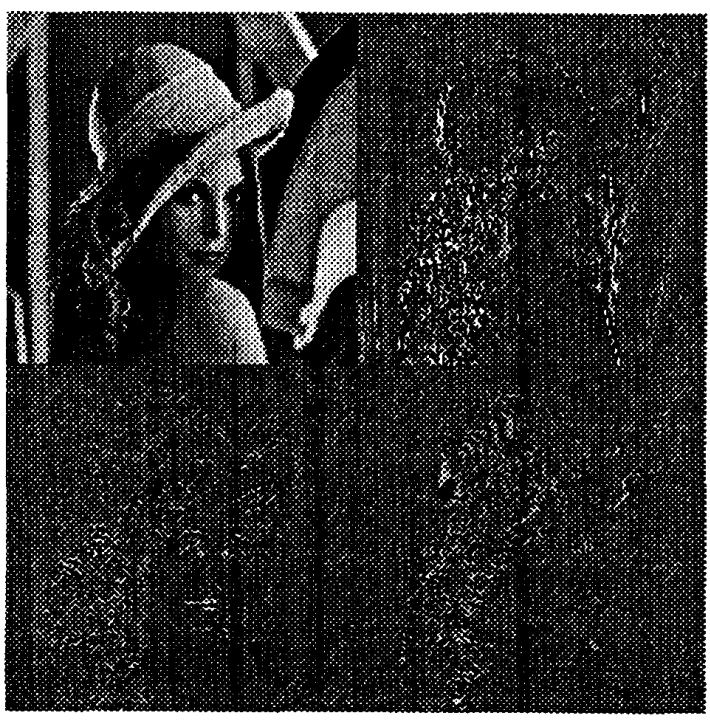

Fig. 5. Linear subband decomposition (QMF filter) 


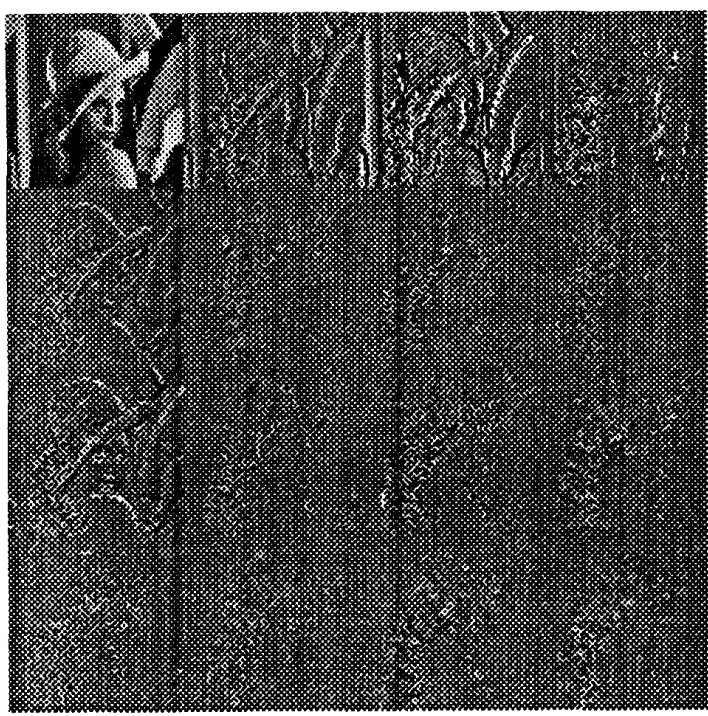

Fig. 6. Morphological subband decomposition (2 iterations)

\section{RESULTS AND CONCLUSION}

When used for coding purposes, the residual images in a multiresolution decomposition are coded by means of some coding technique such as PCM, DPCM or vector quantization. We have coded the residual images using plain PCM quantization to perform the comparison between a linear decomposition using QMF filters and a morphological decomposition with the filters that have been presented.

In the linear case, subband coding schemes benefit from the smaller amplitude resolution of the human visual system to high frequency image components so that a reduced number of quantization levels is used for the high frequency residuals. Bearing in mind the kind of information that can be found in the "small size" residual images of the morphological decomposition, we will apply the same technique. Therefore, it is assumed a poorer amplitude resolution for small size image components and so a coarser quantization is applied to the first obtained residuals.

Coding results are shown in figs. 8 to 11 . As can be seen, for compression ratios around 10 , the perceived qualities are similar for the linear and morphological coding schemes. For larger compressions the quality of the reconstructed image decreases quickly with both schemes. It is worth noticing that the quality of the coded reconstruction is better than the obtained with open-close filters in lossy subsampling schemes [8-10].

The main advantage of the morphological scheme is its reduced computational load. With morphological filtering techniques, only comparisons are performed, whereas the linear filter involves convolution operations. Time comparisons between the linear 8-tap QMF filter

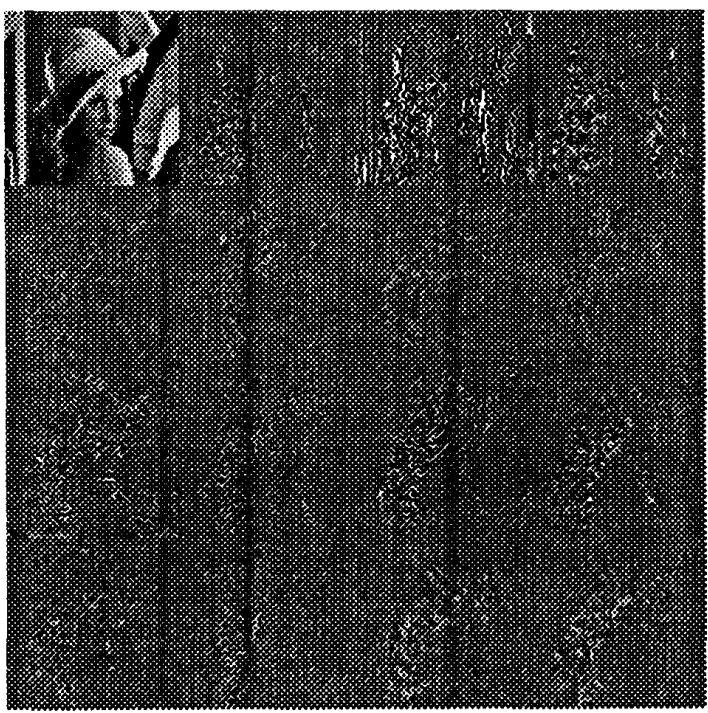

Fig. 7. Linear subband decomposition ( 2 iterations)

and the presented $\xi$ or $\zeta$ filters in a SUN Sparc station have shown that the morphological filtering is performed in only the $20 \%$ of the time of the linear filtering.

In conclusion, a new morphological space-variant filter for lossless subsampling has been presented for a particular downsampling-upsampling strategy. Using this filter and its dual version, a lossless multiresolution decomposition can be computed with very low computational load. Such structures provide a special organization of image data which have proven to be useful for image coding applications, improving the results obtained in previous works also using non-linear filters. If more sophisticated downsampling-upsampling strategies were given, even including geodesic reconstruction to get smoother interpolated images, morphological filters for the simplification step could be designed in a similar way. This would allow improved quality in the progressive image reconstructions but poorer compression results. Two different applications of the presented filters are also under study for video sequence coding: interframe prediction error decomposition and 3D signal decomposition (for the spatial and temporal dimensions). The main point of the these algorithms for video coding is the low computational load when compared to linear subband decomposition schemes.

\section{References}

[1] A. Rosenfeld. Multiresolution Image Processing and Analysis. Springer-Verlag, Berlin 1984.

[2] P.J. Burt, E.H. Adelson. The Laplacian Pyramid as a Compact Image Code. IEEE Trans. on Communications, Vol. COM-31, no. 4, pp. 532-540, April 1983. 
[3] J.W. Woods, S.D. O'Neil. Subband Coding of Images IEEE Trans. on Acoustics, Speech and Signal Processing, Vol. ASSP-34, no.5, pp.1278-1288, October 1986. [4] D. Esteban, C. Galand. Application of quadrature mirror filters to split band voice coding schemes. Proc. of ICASSP-77, pp. 191-195, May 1977.

[5] J. Serra. Image Analysis and Mathematical Morphology. Vol. 2. Chapt. 5-10. Academic Press, London 1988. [6] R.M. Haralick, X. Zhuang, C. Lin, J. Lee. The Digital Morphological Sampling Theorem. IEEE Trans. on ASSP, Vol. ASSP-37 no. 12, pp. 2067- 2090 Dec. 89.

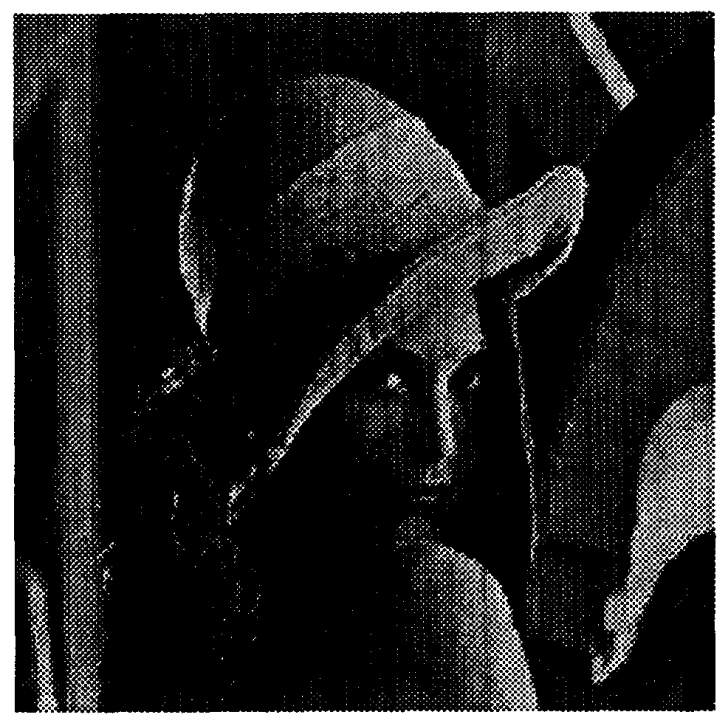

Fig. 8. Morphological subband coded image at $0.65 \mathrm{bpp}$

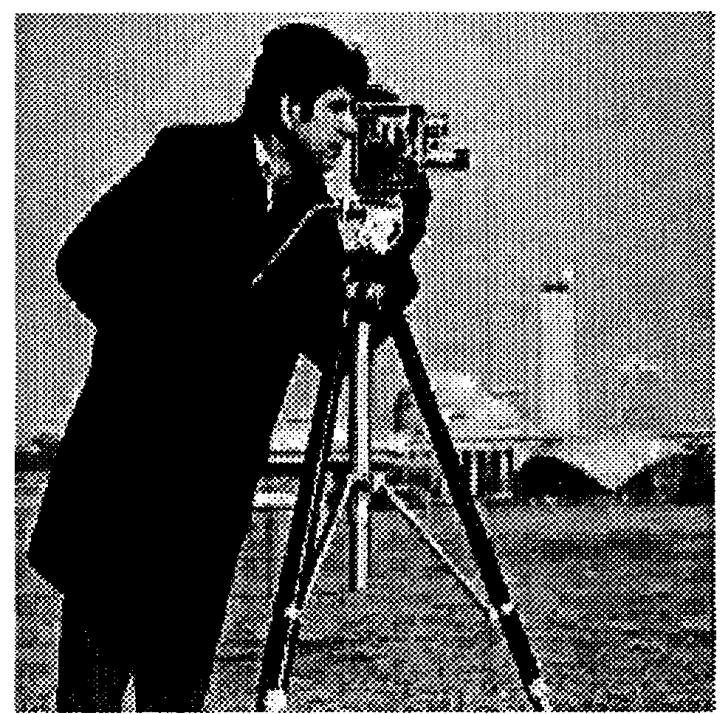

Fig. 10. Morphological subband coded image at $0.8 \mathrm{bpp}$
[7] H. Heijmans, A. Toet. Morphological Sampling. Computer Vision, Graphics and Image Processing: Image Understanding, Vol. 54, no. 3, pp. 384-400, Nov. 1991. [8] A. Toet. A Morphological Pyramidal Image Decomposition. Pattern Recog. Letters 9, pp. 255-261, 1989.

[9] F.K. Sun, P. Maragos. Experiments on Image Compression using Morphological Pyramids. Proc. SPIE VC IP IV, Vol. 1199, pp. 1303-1312. 1989.

[10] S.H. Pei, F.C. Chen. Subband Decomposition of Monochrome and Color Images by Mathematical Mor. phology. Optical Eng. Vol.30 no.7 pp.921-933. July 91.

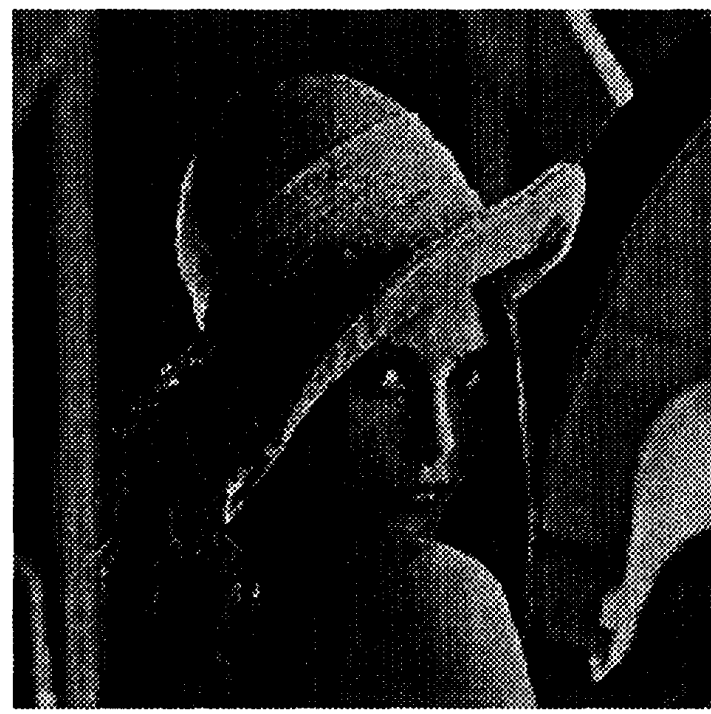

Fig. 9. Linear subband coded image at $0.65 \mathrm{bpp}$

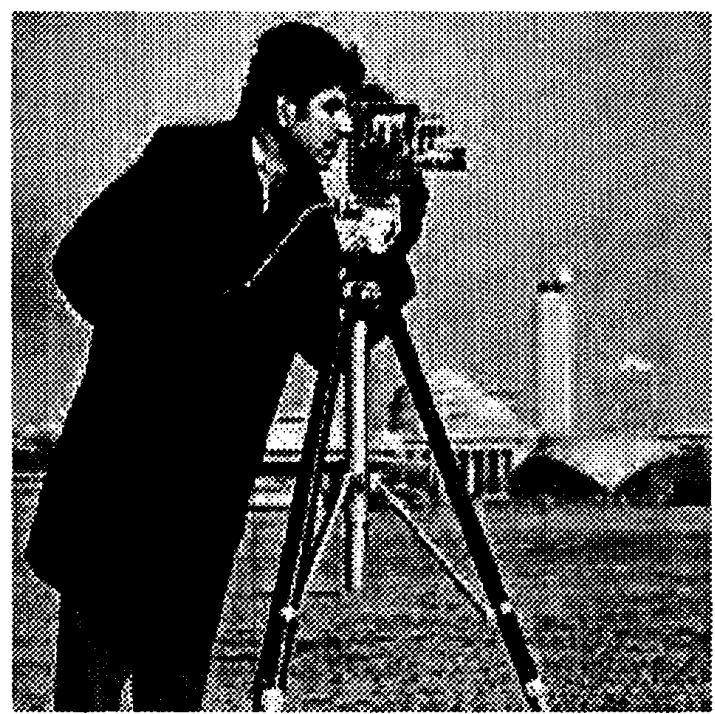

Fig. 11. Linear subband coded image at $0.8 \mathrm{bpp}$ 